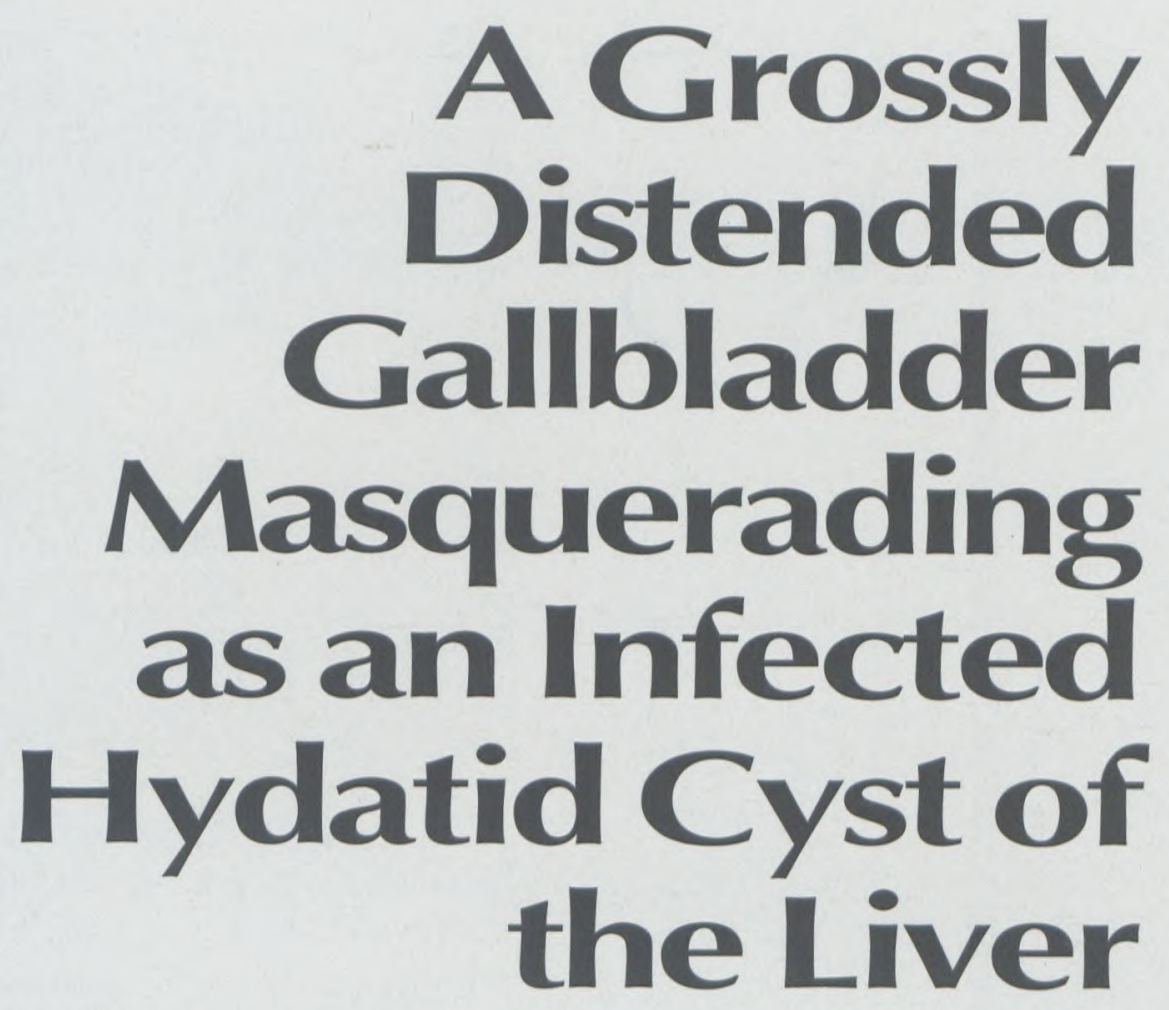

A

WFC van Gelderen

Department of Radiology, Wanganui Base Hospital, Wanganui. New Zealand

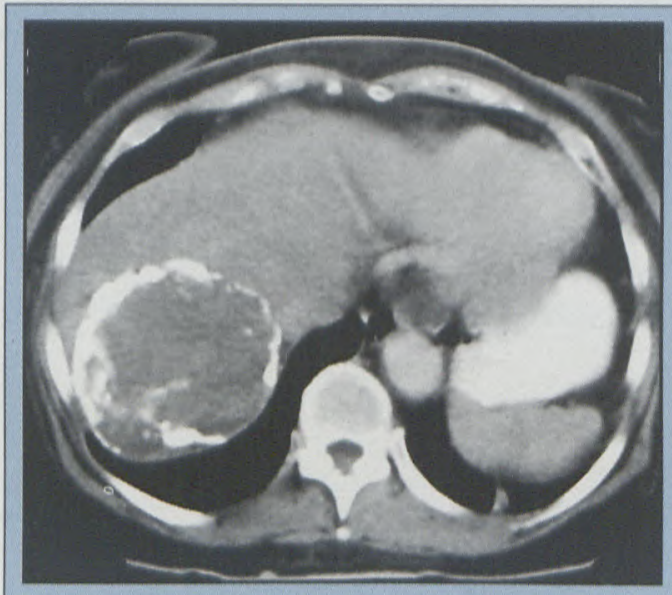

Figure 1: CT demonstrates a heavily calcified hydatid cyst superiorly in the right lobe of the liver. \section{with symptoms of loss of weight, loss of} appetite and nausea. Physical examination revealed a large, non-tender mass in the right side of the abdomen thought to represent a possible cystic kidney. Previous chest radiographs had demonstrated a large calcified hydatid cyst in the right lobe of the liver, as also shown by computed tomography (CT) (Figure 1). Previous barium enema had also demonstrated the large calcified echinococcus cyst and the colon had been markedly displaced to the left due to an infrahepatic mass.

Further CT sections demonstrated an apparently septated, low attenuation mass $10 \mathrm{~cm}$ below the calcified hydatid cyst, in the inferior aspect of the liver. The mass revealed a fairly thick enhancing wall, did not contain any calcification, but demonstrated a small pocket of air in its anterior aspect (Figure 2). The remainder of the liver was normal, but the gallbladder could not be identified.

Subsequent ultrasound (US) examination (Figure 3) confirmed the calcified hydatid cyst. It also demonstrated the hypoechoic mass related to the inferior aspect of the liver, containing multiple small hyperechoic areas, thought to confirm pockets of air as they were almost of "fluorescent" echogenicity and the acoustic shadows cast were not remarkable.

As the gallbladder was not separately identified, a huge distended infected gallbladder was considered in the differential diagnosis, but in view of the other definite echinococcus cyst, a second infected hydatid cyst was advanced as a more likely diagnosis. Militating against either diagnosis was the absence of significant pain or tenderness.

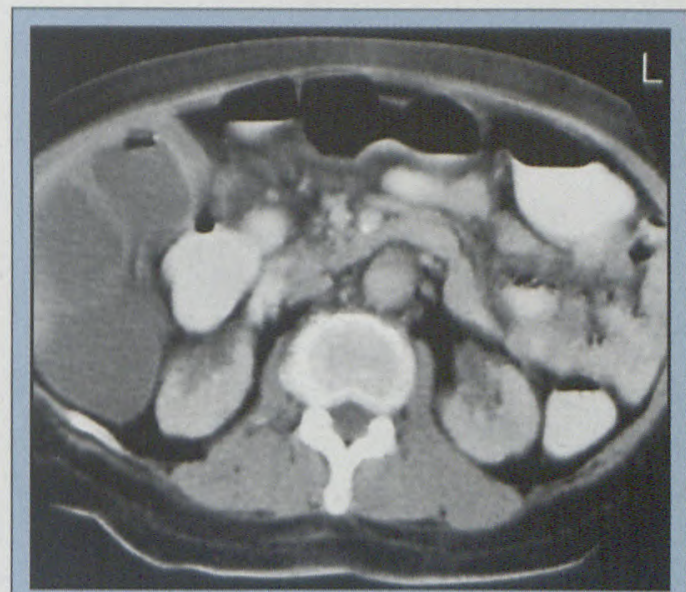

Figure 2: CT section $10 \mathrm{~cm}$ below image in Figure 1. reveals a large septated mass of low attenuation situated at the inferior aspect of the liver containing a pocket of air anteriorly. This was thought to represent an infected hydatid cyst. The gallbladder was not visualised.

A cholecystotomy was performed revealing a grossly distended thick walled gallbladder containing an enormous number and variety of gallstones. Finger palpation of the interior of the gallbladder revealed a probable gallbladder carcinoma and histology after punch biopsy confirmed this diagnosis. 


\section{A Grossly Distended Gallbladder Masquerading as an Infected Hydatid Cyst of the Liver}

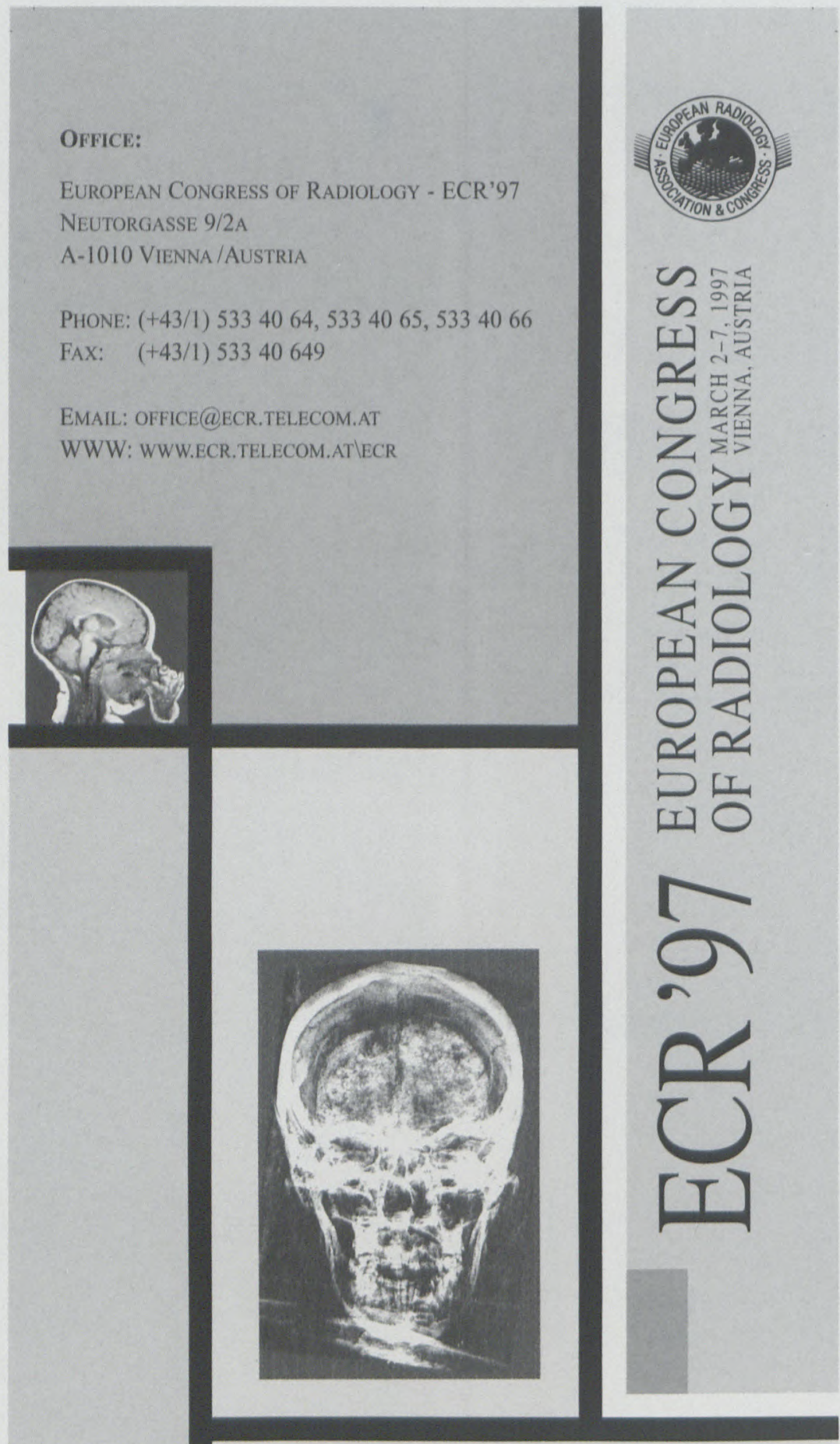

DEADLines:

SUBMISSION OF ABSTRACTS: SEPT. 20, 1996

REDUCED REgistration FEE: DEC. 2, 1996

AdVANCE Registration: Jan. 31, 1997

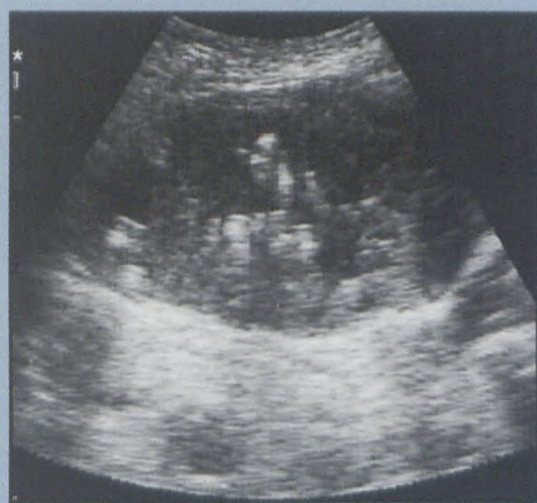

Figure 3: A transverse US section of the mass demonstrates a thick walled structure containing multiple small bright hyperechoic areas casting unremarkable shadows. This was erroneously thought to confirm the CT findings of an infected hydatid cyst.

The surgeon'scomment was asfollows: "None of the many thousands of stones of all varieties and sizes were reported on US or CT scanning, nor was the carcinoma of the gallbladder".

The CT and US images have subsequently been shown to radiological colleagues at other hospitals, and there was some reassurance in that they did not make a correct diagnosis more readily.

The above case report demonstrates just how difficult it can be to establish a correct diagnosis, even if the disease process is of enormous proportions. In retrospect the non-visualisation of the gallbladder on both CT and US should have alerted one, but even then this is a well-known occurrence if the patient had had a meal before the examination. 